\title{
Incidence of ovarian failure in systemic lupus erythematosus after treatment with pulse cyclophosphamide
}

\author{
Elizabeth M McDermott, Richard J Powell
}

\begin{abstract}
Objective-To investigate the incidence of ovarian failure after pulse cyclophosphamide treatment in systemic lupus erythematosus (SLE) and to compare this with two control groups: SLE patients treated with azathioprine, and a healthy age matched population.

Methods-All women patients with SLE treated with pulse cyclophosphamide in our department were identified and questioned concerning menstrual history. All the hospital notes were reviewed and details recorded on dose of cyclophosphamide, duration of treatment, side effects and lowest pretreatment neutrophil and leucocyte counts during the course of treatment. Disease controls were recruited from our department and healthy controls from the local family health services authority (FHSA) register.
\end{abstract}

Results-Incidence of ovarian failure in the premenopausal cyclophosphamide treated group was $54 \%$ and the incidence of premature menopause (occurring before age 40 years) was $41 \%$. Increasing age at start of treatment showed a linear trend with incidence of ovarian failure $(p=0 \cdot 01)$. Using logistic regression, increasing duration of treatment was related to incidence of ovarian failure $(p=0.047$ in those treated age 35 years or younger). An association between the lowest neutrophil count throughout the treatment period, when taken immediately before each planned cyclophosphamide pulse, and the incidence of ovarian failure was also demonstrated $(p=0.04$ in those treated before age 40 years).

Conclusion-Ovarian failure-in particular, premature failure after treatment with pulse cyclophosphamide-is common. Factors associated with increased risk include greater age at start of treatment, longer period of treatment, and greater degree of marrow suppression as assessed by the neutrophil count immediately before each planned cyclophosphamide pulse.

Clinical Immunology Unit, University

Hospital, Nottingham

NG7 2UH,

United Kingdom

E M McDermott

R J Powell

Correspondence to: Elizabeth McDermott.

Accepted for publication 1 December 1995
(Ann Rheum Dis 1996; 55: 224-229)

Cyclophosphamide can induce ovarian failure when used in the treatment of malignancies and inflammatory diseases..$^{1-6}$ Reports of ovarian failure related to cyclophosphamide have varied between $12 \%$ and $83 \% .^{147-9}$ Only three of these studies have included disease controls, and none has incorporated healthy controls. $^{2510}$ Three studies failed to confirm ovarian failure biochemically, ${ }^{578}$ and a further two studies included incomplete hormonal analysis. ${ }^{29}$ Pulse cyclophosphamide with high dose steroids has been shown to be the most effective treatment in lupus nephritis, 571112 and this combination of drugs is also used in the treatment of other features of active systemic lupus erythematosus (SLE) and other connective tissue diseases.

Cyclophosphamide is an alkylating agent that is toxic for both resting and dividing cells because it damages DNA repair mechanisms, ${ }^{13}$ but it causes more damage to the rapidly dividing cells. ${ }^{14}$ Its immunomodulatory mechanism includes suppression of $T$ cell mediated immunity and a reduction of antibody production. ${ }^{15}$

We have therefore investigated the incidence of ovarian failure in patients with SLE treated with pulse cyclophosphamide, and in two control groups: SLE patients with active disease requiring immunosuppressant treatment with azathioprine but never having received cyclophosphamide, and healthy age matched controls. Azathioprine is not recognised to be a cause of ovarian toxicity. ${ }^{16}$ The effects of cumulative dose of cyclophosphamide, age at start of treatment, and use of the oral contraceptive pill during treatment were assessed, as these have previously been implicated as factors in the development of ovarian failure. ${ }^{12}{ }^{17} \mathrm{We}$ also recorded the lowest leucocyte and neutrophil counts immediately before each planned cyclophosphamide pulse, as measures of cytotoxic activity. ${ }^{15}$

\section{Patients and methods}

This retrospective cohort study of patients with SLE, all of whom fulfilled the 1982 revised criteria for classification of SLE, ${ }^{18}$ was approved by the local ethics committee at University Hospital, Nottingham. The cohorts were blinded to the hypothesis before answering the questionnaire. All patients who had been treated with pulse cyclophosphamide within the past seven years in the Clinical Immunological Unit, University Hospital, Nottingham, were identified. Three separate sources were used: pharmacy records, immunosuppressive treatment index cards, and immunology department infusion room diary (in use only over the last five years). Thirty five age matched normal controls for the 
cyclophosphamide treated SLE patients were selected randomly from the normal population from the FHSA register. Twenty consecutive disease controls treated with azathioprine alone over the past seven years comprised the second control group. Each patient was interviewed by one of us (E M), using the same questionnaire that asked about menstrual history, fertility, and menopausal symptoms. Further information was collected on smoking, marital status, occupational status (as a guide to social class and education), racial group, age at menarche, mother's menopause, and parity-all of which have been suggested to influence age at menopause. ${ }^{19-22}$

The menopause was defined as amenorrhoea for at least 12 months, supported by oestradiol concentrations $<150 \mathrm{pmol} / /$ and follicle stimulating hormone (FSH) concentrations $>20 \mathrm{iu} / 1$ on one occasion, ${ }^{23}$ or amenorrhoea for at least six months with the above FSH and oestradiol concentrations on two separate occasions. Premature menopause was defined as a menopause occurring before age 40 years $^{24}$ in the presence of increased FSH and low oestradiol concentrations. Serum FSH and oestradiol concentrations were measured using an immunoradiometric assay (Serono) and radioimmunoassay (Diagnostic Products Ltd), respectively.

Detailed information concerning cyclophosphamide treatment was extracted from the medical notes and checked with the chemotherapy prescription cards. If the patient had been treated previously with cyclophosphamide either in our department or at another hospital, the appropriate notes were reviewed and the treatment details collated. All other immunosuppressant agents were documented from the hospital notes. Immediately before each attendance of the patient for pulse cyclophosphamide treatment, leucocyte and neutrophil counts were recorded. The lowest of these leucocyte/neutrophil counts during the first four weeks of pulse treatment and throughout the whole treatment period were documented. We omitted from the leucocyte analysis those patients whose initial leucocyte count was less than $2 \times 10^{9} / 1$ and then improved shortly after commencement of cyclophosphamide treatment. This was to exclude, as far as possible, disease related leucopenia rather than treatment related leucopenia. Our cyclophosphamide regimen included $1 \mathrm{~g}$ boluses, initially weekly for four weeks, then every two weeks for eight weeks, then monthly for three months. We tailored this regimen individually depending on the patient's response and full blood count, but we usually delayed the bolus if the neutrophil count was less than $1.5 \times 10^{9} /$.

All results were double entered on computer before analysis. Statistical analysis included $\chi^{2}$ test, Fisher's test, logistic regression, and correlation coefficients. Unless otherwise stated, $\chi^{2}$ was used.

\section{Results}

Of the 56 patients treated over seven years with pulse cyclophosphamide, $82 \%$ were identified from more than one source. The 10 recruited from a single source were the patients treated before it became routine to keep a department infusion room diary. Four patients had died during the follow up period: one from septicaemia after four pulses of cyclophosphamide, though she had a normal neutrophil count, and the remaining three of unrelated causes after completion of cyclophosphamide treatment.

Of the remaining 52 patients, 10 had reached their natural menopause before treatment, the ages at last menstrual period being in the range $42-54$ years (mean 48.9 years), excluding one patient with a premature menopause secondary to pituitary disease. A further seven patients had undergone hysterectomy and hence could not accurately date their menopause. Consequently 35 patients, with a documentable date for the last menstrual period, were susceptible to cyclophosphamide induced ovarian failure and 27 of these were younger than 40 years at the start of treatment and could thus also qualify as having a premature menopause. Two patients were unable to assist in the completion of the questionnaire, one because of chronic mental illness requiring institutionalisation, and the other because of poor psychological status. For these two individuals, the hospital and nursing records were used to acquire the menstrual history. The follow up period varied in each patient according to the interval between start of treatment and the questionnaire date, but the minimum was nine months.

Table 1 shows the initial indications for treatment with pulse cyclophosphamide in the study cohort and azathioprine in the control group. None of our patients with lupus nephritis was treated with azathioprine alone, therefore their disease activity was not directly comparable with that of those in the cyclophosphamide group. Of the 35 patients treated with cyclophosphamide, 19 (54\%) subsequently developed ovarian failure. Twelve patients $(63 \%)$ developed amenorrhoea within two years of commencing cyclophosphamide treatment. Of the 27 patients treated before 40 years of age (age range 15-38 years, mean $=28.9), 12(44 \%)$ developed ovarian failure (age range at last menstrual period $20-40$ years, mean 33.5) and in 11 of these the menopause qualified as premature (41\%). Among the cohort who developed a premature menopause, seven (64\%) developed amenorrhoea within two years of the start of their cyclophosphamide treatment. A 28 year old perimenopausal patient denoted by irregular menstruation, menopausal symptoms, and postmenopausal hormone levels, was

Table 1. Indications for treatment with pulse cyclophosphamide (CY) or azathioprine $(A Z A)$ in premenopausal patients with systemic lupus erythematosus between 1987 and 1995

\begin{tabular}{llc}
\hline Indication for treatment & \multicolumn{1}{l}{$C Y$} & \multicolumn{1}{l}{$A Z A$} \\
\hline Vasculitis & $15(42 \cdot 9)$ & $7(35)$ \\
Lupus nephritis & $11(31 \cdot 4)$ & Nil \\
Cerebral lupus & $5(14 \cdot 3)$ & $3(15)$ \\
Systemic symptoms (eg fever) & $4(11 \cdot 4)$ & $10(50)$ \\
\hline
\end{tabular}

Values are number (\%). 
Table 2 Menopause data for patients with systemic lupus erythematosus treated with pulse cyclophosphamide (CY), or azathioprine $(A Z A)$, and normal controls

\begin{tabular}{llll}
\hline & $C Y$ & $A Z A$ & Normal \\
\hline Age (yr) & $\begin{array}{l}36 \cdot 1(17-49) \\
(\mathrm{n}=35)\end{array}$ & $\begin{array}{l}36 \cdot 2(20-50) \\
(\mathrm{n}=20) \\
1(5 \cdot 5)\end{array}$ & $\begin{array}{l}36 \cdot 7(17-49) \\
(\mathrm{n}=35) \\
1(3)\end{array}$ \\
$\begin{array}{c}\text { No reaching } \\
\begin{array}{c}\text { menopause } \\
\text { Age at last } \\
\text { menstrual } \\
\text { period (yr) }\end{array}\end{array}$ & $37 \cdot 8(20-49)$ & 44 & 45 \\
\hline
\end{tabular}

Values are mean (range) or number (\%).

identified, but because her amenorrhoea was of less than six months duration, she was included in the non-ovarian-failure group. The three patients who were taking the oral contraceptive pill at the time of assessment were also included in the menstruating group, though any ovarian failure may have been concealed by withdrawal bleeds. ${ }^{25}$

Table 2 describes the menopausal details of the study cohort and the control groups. In the azathioprine group, doses of the drug ranged from $125 \mathrm{mg}$ to $250 \mathrm{mg}$ (mean $179 \mathrm{mg}$ ). The age ranges and mean age values were similar in all three groups; however, there were no premature menopauses in the azathioprine or healthy control cohorts. Nineteen of the 35 SLE patients developed ovarian failure after cyclophosphamide treatment, in comparison with only one of 18 of the SLE azathioprine controls (two of the original group of 20 having undergone hysterectomy $)(p=0.0005)$ and one of 33 in the healthy control group (two of the original group of 35 also having undergone hysterectomy) $(p=0.000002)$.

Table 3 lists the demographic data of the study cohort and the two control groups. No statistical difference was noted between the menstruating and ovarian failure groups with reference to smoking $(p=0 \cdot 70)$, occupation (professional or skilled compared with semiskilled, unskilled, or unemployed, $p=0.71$ ), disease duration $(p=0.35)$, racial group $(p=0.41)$, or age at menarche $(p=0.99)$. Parity appeared to be greater in the ovarian failure group, but this was not significant $(p=0.07)$. However, marital status (ever married or never married) was significantly different, with a greater number in the ovarian

Table 3 Demographic data of patients with systemic lupus erythematosus treated with pulse cyclophosphamide $(C Y)$ or azathioprine $(A Z A)$, and normal controls

\begin{tabular}{llll}
\hline & $C Y$ & $A Z A$ & Normal \\
\hline Age (yr) & $\begin{array}{l}36 \cdot 1(17-49) \\
(\mathrm{n}=35)\end{array}$ & $\begin{array}{l}36 \cdot 2(20-50) \\
(\mathrm{n}=20)\end{array}$ & $\begin{array}{l}36 \cdot 7(17-49) \\
(\mathrm{n}=35)\end{array}$ \\
$\begin{array}{l}\text { Occupation } \\
\text { Professional/skilled }\end{array}$ & $12(34)$ & $11(55)$ & $6(17)$ \\
$\begin{array}{l}\text { Semi/unskilled } \\
\text { Marital status }\end{array}$ & $23(66)$ & $9(45)$ & $29(83)$ \\
$\quad$ Married & $24(69)$ & $12(60)$ & $25(71)$ \\
$\quad$ Single & $11(31)$ & $8(40)$ & $10(29)$ \\
Menarche (years) & $13 \cdot 2(11-16)$ & $13 \cdot 3(11-15)$ & $13 \cdot 0(11-17)$ \\
Parity & $19(54)$ & $12(60)$ & $26(74)$ \\
Smoking & $10(31)$ & $6(30)$ & $16(49)$ \\
Racial group & & & \\
$\quad$ Afro-Caribbean & $1(6)$ & $4(21)$ & 0 \\
$\quad$ Asian & $2(13)$ & $13(5)$ & 0 \\
$\quad$ White & $11(69)$ & $13(69)$ & $35(100)$ \\
$\quad$ Oriental & $1(6)$ & $1(5)$ & 0 \\
$\quad$ Middle East & $1(6)$ & 0 & 0 \\
Disease duration & $9 \cdot 3(1-20)$ & $10 \cdot 4(1-24)$ & \\
$\quad$ (months) & & & \\
\hline
\end{tabular}

Values are mean (range) or number (\%).
Table 4 Differences in cyclophosphamide (CY) treatment between those who continued to menstruate and those who developed ovarian failure

\begin{tabular}{lcc}
\hline & $\begin{array}{l}\text { Menstruating } \\
\text { group }\end{array}$ & $\begin{array}{l}\text { Ovarian failure } \\
\text { group }\end{array}$ \\
\hline $\begin{array}{l}\text { Duration of treatment } \\
\text { (months) }\end{array}$ & $5 \cdot 63(0.5-14 \cdot 5)$ & $9 \cdot 45(1-28 \cdot 5)$ \\
$\begin{array}{l}\text { Age at start of } \\
\text { treatment (yr) }\end{array}$ & & \\
$<30$ & 8 & \\
$30-39$ & 7 & 9 \\
$\geq 40$ & 1 & 7 \\
$\begin{array}{l}\text { Dose of CY (mg) } \\
\text { Use of the oral } \\
\text { contraceptive pill } \\
\text { during treatment }\end{array}$ & $11750(3000-20500)$ & $16834(3000-65250)$ \\
$\quad$ Yes & & \\
No & 4 & 2 \\
\hline
\end{tabular}

failure group being married $(p=0.03)$. Few subjects could accurately state their mother's age at menopause, making analysis of this factor unreliable.

Table 4 summarises the difference in treatment between those who continued to menstruate and those who developed ovarian failure. Duration of treatment was predictive of development of ovarian failure (logistic regression, $\beta$ coefficient $=0.28, \quad S E=0.14$, $p=0.047)$. For this analysis we excluded patients older than 35 years to limit the effect of the physiological menopause. Those patients treated for more than the usual six months because of continuing active disease were more likely to develop ovarian failure $(p=0.067)$. Age at start of cyclophosphamide treatment and incidence of ovarian failure showed a linear trend with increasing age $\left(\chi^{2}\right.$ for linear trend, $p=0 \cdot 01)$. However, there was no correlation between age at the start of pulse cyclophosphamide and treatment duration $(r=-0 \cdot 17,95 \% \mathrm{CI}-0.48$ to $0 \cdot 17)$. The mean dose of pulse cyclophosphamide was greater in the group who developed ovarian failure than in the menstruating group, though this did not reach statistical significance $(p=0 \cdot 24)$. There was no significant difference in mean total dose of cyclophosphamide between the $<30,30-39$ or $\geq 40$ year age groups $(p=0 \cdot 29)$.

Factors other than cyclophosphamide influencing the development of ovarian failure were also analysed. Although toxic effects such as vomiting, alopecia, and fatigue appeared to be greater in the group who developed ovarian failure, no statistical association was observed (eight of 15 and 12 of 19 , five of 15 and eight of 19 , one of 14 and two of 17 , respectively). There was no significant difference in concurrent usage of methylprednisolone, methotrexate, azathioprine, or chlorambucil between the group developing amenorrhoea and those who continued to menstruate. Furthermore, we found no association between ovarian failure and oral contraceptive pill usage during cyclophosphamide treatment (Fisher's exact test: $p=0.37$ ) (table 4).

Disturbance of menstruation (change in menstrual pattern compared with that before treatment) during cyclophosphamide treatment appeared greater in the patients who subsequently developed ovarian failure (14 of $18(78 \%))$ compared with those who did not (seven of $15(47 \%)$ ), but this did not reach 
statistical significance $(p=0 \cdot 06)$. However, a significant relationship between cessation of menstrual periods for more than four months during treatment and subsequent development of ovarian failure was established: two of 15 compared with 10 of $18(p=0 \cdot 01)$.

The lowest neutrophil counts recorded throughout the treatment period, when taken immediately before the next planned pulse of cyclophosphamide, were split into low $\left(\leqslant 1.5 \times 10^{9} / 1\right)$ and high groups. In those treated before 40 years of age, low neutrophil counts were associated with increased ovarian failure $(p=0.04)$. In the whole cohort, $70 \%$ of these lowest 'pretreatment' neutrophil counts were less than $2 \times 10^{9} / 1$ (mean $=1.9 \times 10^{9} / 1$, range $0.05-9 \cdot 6)$, indicating that sufficient quantities of cyclophosphamide were used to induce neutropenia. No significant correlation was established between the total cyclophosphamide dose and the lowest leucocyte count taken before each planned cyclophosphamide pulse $(r=-0.31,95 \% \mathrm{CI}-0.59$ to 0.05$)$.

Within the group who continued menstruating after cyclophosphamide treatment, only three patients wished to conceive and two of these were successful: one experienced an ectopic pregnancy and conceived after a further 15 months, and the other became pregnant after only three months. The single patient who failed to conceive had been infertile for 15 years before cyclophosphamide treatment, despite regular unprotected intercourse.

\section{Discussion}

The study revealed a $41 \%$ incidence of premature ovarian failure in the SLE group treated with cyclophosphamide before age 40 years. Our results are similar to those of Austin et al, who found an ovarian failure rate of $45 \%$ in patients younger than 45 years treated with intravenous pulse cyclophosphamide; ${ }^{5}$ however, their study lacked the confirmation of the menopause by, appropriate hormone assays. Lower incidences of ovarian failure have been reported by Wang et al, ${ }^{1}$ namely $27 \%$ after treatment with oral cyclophosphamide, and by Boumpas et $a l_{,}^{2}$ who reported an incidence of $19 \%$ in patients treated for six months and $38 \%$ in those treated for 30 months. None of the above studies included a normal control population. One reason why incidences of ovarian toxicity have varied widely between studies is that there is no accepted cyclophosphamide treatment regimen, so treatment durations and mean doses differ. The study by Austin's group had a median follow up period of seven years, which is much longer than the median follow up of 36 months in our study. We showed that $64 \%$ of documented premature ovarian failures occurred within two years of the start of treatment so, considering the minimum follow up time of only nine months, it is likely that an even greater number of patients will subsequently develop premature ovarian failure. We classified the perimenopausal 28 year old patient as 'premenopausal' because menopausal status is assessed more accurately by menstrual history than by hormone levels during the perimenopause; ${ }^{26}$ however, it is very likely that she will develop a premature menopause in the future.

Each ovary has a reservoir of primordial follicles and from these a small number of follicles start to develop during the early luteal phase of each menstrual cycle and continue to mature for about 60 days until the late luteal phase two cycles later. ${ }^{27}$ These primary follicles, about 20 per ovary, have increased sensitivity to stimulation by $\mathrm{FSH}$, and at this stage are most sensitive to cyclophosphamide toxicity. This has been confirmed in animal models by Ataya et al, ${ }^{28}$ who compared the size of follicles in the ovaries of rats treated with cyclophosphamide and controls. They showed that at the end of treatment there was no difference in the number of small (primordial) follicles between the two groups, but a significant reduction in the number of larger (primary) follicles in the treated rats. When a maturing cohort of primary follicles is damaged by cyclophosphamide, oestrogen production decreases, with a consequent increase in pituitary gonadotrophin concentrations. The greater the damage to the developing follicles by the cyclophosphamide, the greater the resulting hormonal imbalance and hence disruption of the normal cyclical pattern of menstruation. In our study, amenorrhoea for more than four months during treatment resulted in a significantly increased risk of ovarian failure. Thus menstrual history may be useful as a prognostic guide to the subsequent development of ovarian failure. The increase in FSH concentrations after follicular damage stimulates a new cohort of primordial follicles to develop, which enhances their sensitivity to the toxic effects of cyclophosphamide. Although it has previously been suggested as a method for ovarian protection, ${ }^{7}$ timing pulses with the menstrual cycle is likely to be ineffectual, because the vicious cycle of follicular destruction continues throughout the duration of cyclophosphamide treatment. Consequently, the longer the duration of treatment, the greater the number of continually developing follicles that are exposed to damage.

We confirmed that the duration of cyclophosphamide treatment was a significant factor in the development of ovarian failure in those treated at age 35 years or younger. We excluded from this particular analysis patients older than 35 years at the start of their treatment, because treatment duration extended up to 28.5 months, and the follow up period was of up to seven years, and we wanted to exclude the effect of the natural menopause, which normally occurs between ages 45 and $55 .{ }^{26}$ In animal studies, both the duration of treatment and the dosage of cyclophosphamide have been shown to be important in the development of ovarian failure. ${ }^{28}$ In our study, though the total dose of cyclophosphamide was not significantly associated with ovarian failure, doses tended to be greater in the ovarian failure group.

In addition to the dose related bone marrow suppression that is a feature of all cytotoxic drugs, ${ }^{29}$ cyclophosphamide is reported to have 
immunomodulatory effects on both $\mathrm{T}$ cells and B cells. ${ }^{15}$ The degree of immunosuppression denoted by the lowest neutrophil count taken immediately before each planned cyclophosphamide pulse was associated with the development of ovarian failure. Patients aged 40 years or more were excluded from this analysis, in view of the duration of their follow up and the effect of the natural menopause. If neutropenia is necessary to the therapeutic effect of cyclophosphamide in SLE, then ovarian toxicity appears to be an inevitable consequence of this treatment.

There was a trend of increasing incidence of ovarian failure in the $<30$ year group, 30-39 year group, and those $\geq 40$ years, respectively. This finding is in agreement with those of a study using oral cyclophosphamide that also demonstrated a continuous age related trend. ${ }^{1}$ The size of the follicular reserve is the major determinant of transition into perimenopause and subsequent menopause, ${ }^{23}$ and it has been suggested that, because the pool of primordial follicles is smaller in older women, they have diminished reserves and therefore develop ovarian failure sooner. ${ }^{12}$ In fact, there is a continued logarithmic decrease in follicle numbers from the maximum number in the seven month old fetus until the last decade before the menopause, when numbers decline more dramatically. ${ }^{23}$ Mean FSH concentrations start to increase in the decade immediately before the menopause in all women. ${ }^{30}$ Richardson proposed that this increased concentration of FSH caused an increased number of primordial follicles to develop each month, and hence a greater number ultimately to suffer follicular atresia. ${ }^{23}$ If this is true, the increased risk of ovarian failure in the older patients treated with cyclophosphamide can be explained by the increased number of developing cyclophosphamide sensitive follicles.

The duration of the follicular recruitment and development cycle, and hence the period of susceptibility to damage, are central to the development of ovarian toxicity. If the cycle of follicular development could be slowed or stopped by medication, the ovary may be protected. The oral contraceptive pill, progesterone, and gonadotrophin releasing hormone analogues $(\mathrm{GnRH})$ have all been suggested to have protective effects on the ovary. ${ }^{173132}$ The oral contraceptive pill inhibits ovulation via a negative feedback mechanism on the hypothalamus, reducing the surge of $\mathrm{FSH}$ and luteinising hormone (LH) and preventing ovulation; however, follicular development may continue, particularly with the newer lower dose oestrogen pills. In our study, we found that the low dose oral contraceptive pill failed to protect the ovaries. Chapman's small study of six women did show a protective effect of the higher dose oral contraceptive pill (ethinyl oestradiol $50 \mu \mathrm{g}$ ), but this dose is now rarely used because of the increased risk of thrombosis. ${ }^{17}$ In a single study, progesterone has been shown to protect fertility in rats treated with cyclophosphamide; ${ }^{31}$ however, there is no evidence that it prevents the occurrence of premature menopause and, because it acts mainly on the uterus, preparing it for implantation, it is unlikely to affect ovarian function. ${ }^{33} \mathrm{~A}$ novel approach would be to use $\mathrm{GnRH}$ analogues which initially stimulate but then suppress pituitary gonadotrophin release with continued administration. The decrease in production of $\mathrm{LH}$ and $\mathrm{FSH}$ reduces follicular development, presumably with consequent reduction in ovarian sensitivity to cyclophosphamide. $\mathrm{GnRH}$ analogues are currently used in the treatment of advanced breast cancer, prostatic cancer, and endometriosis. ${ }^{34}$ Ataya $e t$ al showed a protective effect on the ovaries of long term LH releasing hormone agonist in cyclophosphamide treated rats, ${ }^{32}$ but as yet there have been no trials in humans. GnRH analogues increase ovarian oestradiol release for three days after administration, before slowly decreasing over the next two weeks. In patients with SLE, this oestradiol surge may have an adverse influence on disease activity: worsening of SLE associated thrombocytopenia in a patient treated with the GnRH analogue, buserelin, for endometriosis has recently been reported. ${ }^{35}$ In contrast, clinical improvement in four of six patients with active SLE treated with GnRH in addition to steroids and immunosuppressants has been reported. ${ }^{36} \mathrm{~A}$ significant problem with $\mathrm{GnRH}$ analogues in SLE may be loss of bone mineral density. It has been shown that, after only four months of treatment with the $\mathrm{GnRH}$ analogue, goserelin, bone mineral density in women was reduced by $3.7 \%{ }^{37}$ In patients with SLE, who may already be at risk from osteoporosis as a result of concomitant steroid treatment, this is an important consideration, though the effect may be reduced by hormone replacement therapy. ${ }^{37}$ If GnRHs are safe in SLE, they could be used with cyclophosphamide treatment for the dual purpose of ovarian protection and reduction of disease activity. Storage of ova is becoming available, and for those wishing to start a family after cyclophosphamide treatment this may be helpful. ${ }^{38}$ However, ova storage takes time to organise, and often the decision to commence treatment cannot be delayed, because vital organs are in danger.

We used a control group of patients with active SLE also requiring immunosuppression to observe the effect of active disease on the timing of the menopause. The incidences of ovarian failure in the azathioprine treated control group and the healthy control group were very similar, suggesting that active disease does not significantly affect age at menopause. It is acknowledged that the degree of disease activity in the azathioprine group may have been less than that in the cyclophosphamide group. However, the group of SLE patients who had reached their menopause before commencement of cyclophosphamide treatment had a mean age at menopause of 48.9 years, similar to the estimated mean for Western Europe (49-50 years). ${ }^{24}$

Confounding factors, such as smoking and occupation, were not significantly different between the group that continued to menstruate and the group that developed ovarian failure, but marital status did differ: ever being 
married was more common in the group who subsequently developed ovarian failure, contrary to expectation, as ever being married is usually associated with later onset of menopause. ${ }^{21}$ As the mean age was greater in the ovarian failure group, it could simply be that older women are more likely to have married. Likewise, parity appeared to be more common in the ovarian failure group, despite an association with later age at menopause, ${ }^{21}$ but this may also have been a consequence of the age difference. Hence the incidence of ovarian failure in our study group cannot be explained by confounding factors. Demographic data of the three groups were similar, except for race in the healthy control group. These control subjects were randomly selected from a population of more than 600000 from the Nottingham area, which is well represented by whites, asians, and Afro-Caribbeans. This discrepancy in race between the diseased and healthy groups emphasises the increased prevalence of SLE in Afro-Caribbean groups. ${ }^{39}$

In conclusion, the risk of cyclophosphamide induced menopause in women with SLE is considerable. In our study, the incidence was $54 \%$ in all age groups and $44 \%$ in those treated before the age of 40 years, whilst $41 \%$ actually had a premature ovarian failure. Of the risk factors considered, duration of treatment and 'pretreatment' neutropenia appeared to be important. Older age at start of cyclophosphamide treatment also increased the risk of developing ovarian failure. These findings underline the necessity for discussion concerning ovarian toxicity in all premenopausal women before commencement of cyclophosphamide treatment.

1 Wang C L, Wang F, Bosco J J. Ovarian failure in oral cyclophosphamide treatment for systemic lupus erythematosus. Lupus 1995; 4: 11-4

2 Boumpas D T, Austin H A, Vaughn E M, Yarboro C H, Klippel J H, Balow J E. Risk for sustained amenorrhoea in patients with systemic lupus erythematosus receiving in patients with systemic lupus erythematosus receiving intermittent pulse cyclo

3 Chapman R M, Sutcliffe S B, Malpass J S. Cytotoxicinduced ovarian failure in women with Hodgkin's disease. ҰAMA 1979; 242: 1171-81.

4 Warne G L, Fairley K F, Hobbs J B, Martin F I R. Cyclophosphamide-induced ovarian failure. $N \mathrm{Engl} f \mathrm{Med}$ 1973; 289: 1159-62.

5 Austin H A, Klippel J H, Balow J E, et al. Therapy of lupus nephritis. Controlled trial of prednisolone and cytotoxic drugs. $N$ Engl F Med 1986; 314: 614-9.

6 Sanders J E, Buckner C D, Amos D, et al. Ovarian function following marrow transplantation for aplastic anaemia or leukaemia. F Clin Oncol 1988; 6: 813-8.

7 Belmont H M, Storch M, Buyon J, Abramson S. New York University/Hospital for Joint Diseases experience with intravenous cyclophosphamide treatment: efficacy in intravenous cyclophosphamide treatment: efficacy in
steroid unresponsive lupus nephritis. Lupus 1995; 4: steroid $104-8$.

8 Langevitz P, Klein L, Pras M, Many A. The effect of cyclophosphamide pulses on fertility in patients with lupus nephritis. Am $\mathcal{F}$ Reprod Immunol 1992; 28: 157-8.

9 Uldall P R, Kerr D N S, Tacchi D. Sterility and cyclophosphamide. Lancet 1972; 1: 693-4.

10 Koyama H, Wada T, Nishizawa Y, et al. Cyclophosphamide-induced ovarian failure and its therapeutic significance in patients with breast cancer. Cancer 1977; 39: 1403-9.

11 Steinberg A D, Decker J L. A double-blind controlled tria comparing cyclophosphamide, azathioprine and placebo in the treatment of lupus glomerulonephritis. Arthritis Rheum 1974; 17: 923-37.

12 Decker J L, Klippel J H, Plotz P H, Steinberg A D. Cyclophosphamide or azathioprine in lupus glomerulonephritis: a controlled trial: results at 28 months. Ann Intern Med 1975; 83: 606-15.

13 Luqmani R A, Palmer R G, Bacon P A. Azathioprine, cyclophosphamide and chlorambucil. Bailliere's Clin Rheumatol 1990; 4: 595-619.

14 Clayton P E, Shalet S M, Price D A. Gonadal function after chemotherapy and irradiation for childhood malignancies. Horm Res 1988; 30: 104-10.

15 Fox D A, McCune W J. Immunosuppressive drug therapy of systemic lupus erythematosus. Rheum Dis Clin North Am 1994; 20: 265-99.

16 Roubenoff R, Hoyt J, Petri M, Hochberg M C, Hellmann D B. Effects of antiinflammatory and immunosuppressive drugs on pregnancy and fertility. Semin Arthritis Rheum 1988; 18: 88-110.

17 Chapman R M, Sutcliffe S B. Protection of ovarian function by oral contraceptives in women receiving chemotherapy for Hodgkin's disease. Blood 1981; 58: 849-51.

18 Tan E M, Cohen A S, Fries J F, et al. The 1982 revised criteria for the classification of systemic lupus erythematosus. Arthritis Rheum 1982; 25: 1271-7.

19 Mattison D. Effects of smoking on fertility from gametogenesis to implantation. Environ Res 1982; 28: 410-33.

20 Brambilla D J, McKinlay S M. A prospective study of factors affecting age at menopause. $\mathcal{F}$ Clin Epidemiol 1989; 42: $1031-9$.

21 Stanford J L, Hartge P, Brinton L A, Hoover R N, Brookmeyer $R$. Factors influencing the age at natural menopause. F Chron Dis 1987; 40: 995-1002.

22 Whelan E A, Sandler D P, McConnaughey D R, Weinberg C R. Menstrual and reproductive characteristics and age at natural menopause. Am $\mathcal{F}$ Epidemiol 1990; 131: $625-32$.

23 Richardson S J. The biological basis of the menopause. Balliere's Clin Endocrinol Metab 1993; 7: 1-16.

24 Symonds $E$ M, ed. The changes of the menstrual cycle. In: Essential obstetrics and gynaecology, 2nd edn, chapter 21. Edinburgh: Churchill Livingstone, 1992, 209-17.

25 Anderson M, Braude P, Cardoza L, et al. Contraception and sterilization. In: Chamberlain G V P, ed. Gynaecology by Ten Teachers, 14th edn, chapter 11, part iii. London: Edward Arnold, 1986; 242-3.

26 Metcalfe M G. The approach of menopause: a New Zealand study. $N Z$ Med F 1988; 101: 103-6.

27 Genuth S M. The reproductive glands. In: Berne $R M$ Levy M N, eds. Physiology, chapter 51. Missouri: Mosby Year Book, 1993; 1006-7.

28 Ataka K, Moohissi K. Chemotherapy induced premature ovarian failure: mechanisms and prevention. Steroids 1989; 54: 607-26.

29 Clements P J, Davies J. Cytotoxic drugs: their clinical application to the rheumatic diseases. Semin Arthritis Rheum 1986; 15: 231-53.

30 Lee S J, Lenton E A, Sexton L, Cooke I D. The effect of age on the cyclical patterns of plasma $\mathrm{LH}, \mathrm{FSH}$, oestradiol and progesterone in women with regular cycles. Hum Reprod 1988; 3: 851-5.

31 Montz F J, Wolff A J, Gambone J C G. Gonadal protection and fecundity rates in cyclophosphamide treated rats. Cancer Res 1991; 51: 2124-6.

32 Ataya K M, McKanna J A, Weintraub A M, Clark M R, LeMarie W J. Luteinizing hormone releasing hormone agonist for the prevention of chemotherapy induced ovarian follicular loss in rats. Cancer Res 1985; 45: 3651-6.

33 Llewellyn-Jones $D$, ed. The menstrual cycle and menstruation. In: Fundamentals of obstetrics and gynaecology, 4th edn, chapter 5. London: Faber and Faber, 1986; 50-60.

34 Miller R M, Frank R A. Zoladex (goserelin) in the treatment of benign gynaecological disorders: an overview of safety and efficacy. Br 7 Obstet Gynaecol 1992; 99: 37-41.

35 Miyagawa S, Shirai T, Shimamoto I, Ichijo M, Ueki H. Worsening of systemic lupus erythematosus-associated thrombocytopenia after administration of gonadotrophinreleasing hormone analog. Arthritis Rheum 1994; 37: releasing

36 Catania A, Mangone I, Motta P, Zanussi C. Administration of gonadotrophin-releasing analog as adjuctive therapy in women with systemic lupus erythematosus. Arthritis Rheum 1989; 32: 1186-8.

37 Chris P, Goa K L. Goserelin: a review of its pharmacodynamic and pharmacokinetic properties and clinical use in sex hormone-related conditions. Drugs 1991; 2: 254-88.

38 Baber R, Abdalla H, Studd J. The premature menopause. In: Studd J, ed. Progress in obstetrics and gynaecology, Vol 9, chapter 13. London: Churchill Livingstone, 1991

Hopkinson N D, Doherty M, Powell R J. The prevalence and incidence of systemic lupus erythematosus in Nottingham, UK, 1989-1990. Br f Rheumatol 1993; 32: $110-5$. 